\title{
Quality assessment of graphene: Continuity, uniformity, and accuracy of mobility measurements
}

Mackenzie, David; Buron, Jonas Christian Due; Whelan, Patrick Rebsdorf; Caridad, Jose; Bjergfelt, Martin; Luo, Birong; Shivayogimath, Abhay; Smitshuysen, Anne Lyck; Thomsen, Joachim Dahl; Booth, Tim

Total number of authors:

15

Published in:

Nano Research

Link to article, DOI:

10.1007/s12274-017-1570-y

Publication date:

2017

Document Version

Peer reviewed version

Link back to DTU Orbit

Citation (APA):

Mackenzie, D., Buron, J. C. D., Whelan, P. R., Caridad, J., Bjergfelt, M., Luo, B., Shivayogimath, A.,

Smitshuysen, A. L., Thomsen, J. D., Booth, T., Gammelgaard, L., Zultak, J., Jessen, B. S., Bøggild, P., \& Petersen, D. H. (2017). Quality assessment of graphene: Continuity, uniformity, and accuracy of mobility measurements. Nano Research, 10(10), 3596-3605. https://doi.org/10.1007/s12274-017-1570-y

\section{General rights}

Copyright and moral rights for the publications made accessible in the public portal are retained by the authors and/or other copyright owners and it is a condition of accessing publications that users recognise and abide by the legal requirements associated with these rights.

- Users may download and print one copy of any publication from the public portal for the purpose of private study or research.

- You may not further distribute the material or use it for any profit-making activity or commercial gain

- You may freely distribute the URL identifying the publication in the public portal 

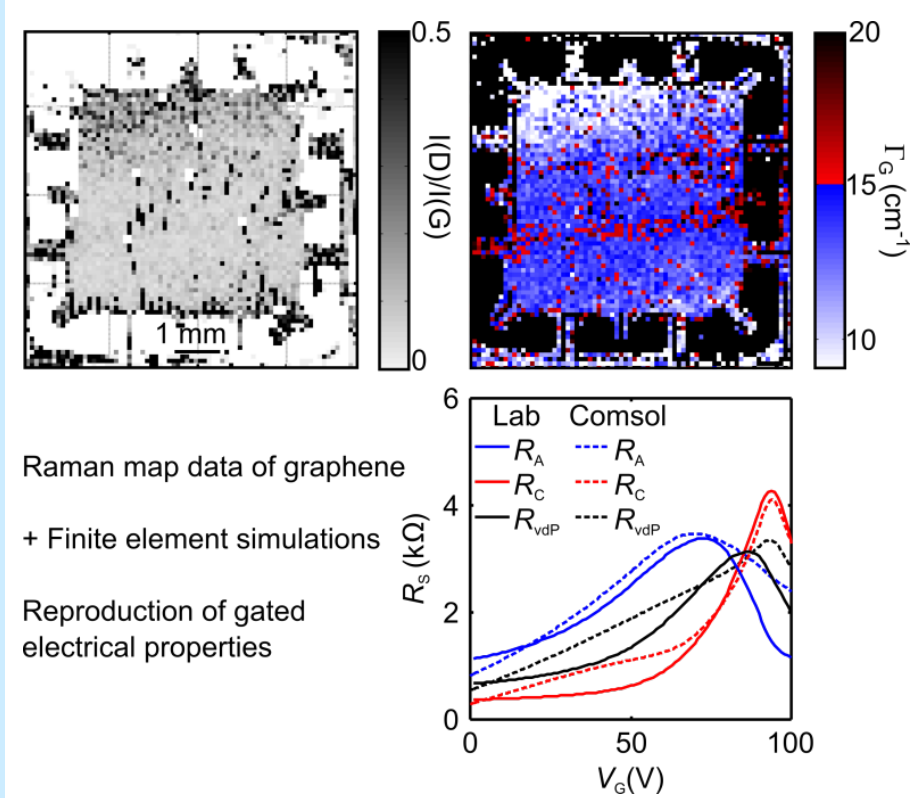

We present a simple framework for assessing both the homogeneity of large-area graphene devices and the accuracy of electrical measurements. Finite element simulations and electrical measurements show that non-uniform doping in graphene can lead to systematic overestimation of the field-effect mobility and other electrical parameters, which can be corrected using the presented methods. 


\title{
Quality assessment of graphene: Continuity, uniformity, and accuracy of mobility measurements
}

David M. A. Mackenzie ${ }^{1,2}(\bowtie)$, Jonas D. Buron ${ }^{1,2}$, Patrick R. Whelan ${ }^{1,2}$, José M. Caridad ${ }^{1,2}$, Martin Bjergfelt $^{1,2}$, Birong Luo ${ }^{1,2}$, Abhay Shivayogimath ${ }^{1,2}$, Anne L. Smitshuysen ${ }^{1,2}$, Joachim D. Thomsen ${ }^{1,2}$, Tim J. Booth ${ }^{1,2}$, Lene Gammelgaard $^{1,2}$, Johanna Zultak ${ }^{1,2}$, Bjarke S. Jessen ${ }^{1,2}$, Peter Bøggild ${ }^{1,2}$, and Dirch H. Petersen ${ }^{1,2}$

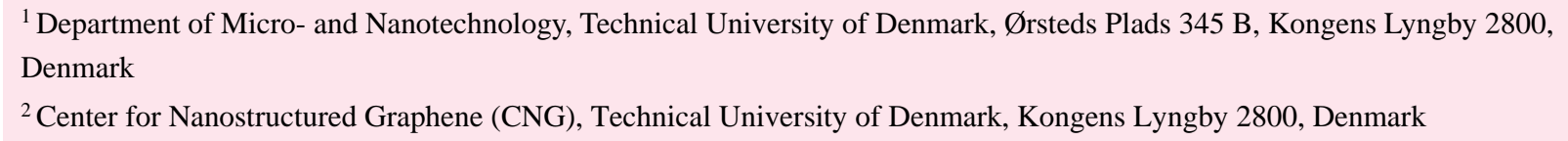

\begin{abstract}
With the increasing availability of large-area graphene, the ability to rapidly and accurately assess the quality of the electrical properties has become critically important. For practical applications, spatial variability in carrier density and carrier mobility must be controlled and minimized. We present a simple framework for assessing the quality and homogeneity of large-area graphene devices. The field effect in both exfoliated graphene devices encapsulated in hexagonal boron nitride and chemical vapor-deposited (CVD) devices was measured in dual current-voltage configurations and used to derive a single, gate-dependent effective shape factor, $\beta$, for each device. $\beta$ is a sensitive indicator of spatial homogeneity that can be obtained from samples of arbitrary shape. All 50 devices investigated in this study show a variation (up to tenfold) in $\beta$ as a function of the gate bias. Finite element simulations suggest that spatial doping inhomogeneity, rather than mobility inhomogeneity, is the primary cause of the gate dependence of $\beta$, and that measurable variations of $\beta$ can be caused by doping variations as small as $10^{10} \mathrm{~cm}^{-2}$. Our results suggest that local variations in the position of the Dirac point alter the current flow and thus the effective sample shape as a function of the gate bias. We also found that such variations lead to systematic errors in carrier mobility calculations, which can be revealed by inspecting the corresponding $\beta$ factor.
\end{abstract}

\section{KEYWORDS}

CVD graphene, doping inhomogeneity, electrical measurements, van der Pauw, hBN-encapsulated graphene, finite element simulations, Raman mapping

\section{Introduction}

Monolayer graphene is transitioning from the laboratory to the marketplace, largely as a result of the development of fast and reliable wafer-scale and roll-to-roll growth techniques. However, most applications require narrow tolerance in the

Address correspondence to dmac@nanotech.dtu.dk 
electrical parameters, which must be reliably monitored and controlled if graphene is to be successfully commercialized. Therefore, there is a fundamental need for consistent and accurate electrical measurements to match the increasing throughput of graphene samples grown by chemical vapor deposition (CVD). Typical transport measurement methods currently in use rely on relatively slow lithographic techniques [1, 2], microscopic four-point probes requiring physical contact with the graphene film [3], or contactless measurements where electrical parameters are obtained directly (as in terahertz time-domain spectroscopy) [4] or indirectly (as in Raman spectroscopy) [5].

Here, using electrical measurements performed in orthogonal dual-electrode configurations and finite element simulations, we systematically investigate doping anisotropies in wafer-scale CVD graphene. We propose a methodology capable of evaluating the impact of the non-uniformity of particular sample or a set of samples, and show how non-uniform doping can lead to a systematic overestimation of the field-effect mobility.

\section{Methods}

Figure 1 shows a schematic of the fabrication of millimeter-scale devices based on large-scale CVD graphene. Figure 1(a) shows a section of the device wafer, where single-layer graphene was transferred on top of a silicon wafer with a 165-nm insulating $\mathrm{Si}_{3} \mathrm{~N}_{4}$ layer using the wet polymethylmethacrylate (PMMA) transfer method (device type A) [6] or onto a 300-nm $\mathrm{SiO}_{2}$ layer using the $\mathrm{NaOH}$ transfer method (device type B) [7]. Figure 1(b) shows the deposition of metal electrodes ( $5 \mathrm{~nm} \mathrm{Cr}, 45 \mathrm{~nm} \mathrm{Au}$ ) through a shadow mask using a Wordentec QCL 800 electron-beam evaporation system. Figure 1(c) shows the device definition via selective laser ablation of graphene [8]. This task was performed using a 3D-Micromac AG microSTRUCT vario laser micromachining system, which ablates the graphene without damaging the substrate [8] or affecting its electrical properties [9]. The resulting square device shown in Fig. 1(d) has a central area of $5 \mathrm{~mm} \times 5 \mathrm{~mm}$.

The present small-scale, high-quality devices (type C) were prepared using high-quality graphene fully encapsulated within hexagonal boron nitride $(\mathrm{hBN})$ as control samples for comparison with CVD graphene. These devices were fabricated using a temperature-controlled dry transfer (hot-pickup) approach, described in detail in Ref. [10]. Figure 2(a) shows the fabricated hBNgraphene-hBN stack before patterning. This stack was patterned into $10 \mu \mathrm{m} \times 10 \mu \mathrm{m}$ square devices by electron-beam lithography (EBL), as shown in Fig. 2(b), using $\mathrm{SF}_{6}$ and $\mathrm{O}_{2}$ plasma etching to shape the stack (Fig. 2(c)) [10]. Then, a standard EBL step is performed to define $5 \mathrm{~nm} \mathrm{Cr} / 30 \mathrm{~nm}$ Au electrodes (Fig. 2(d)). This type of device typically shows higher carrier mobilities compared to CVD samples $\left(>20,000 \mathrm{~cm} \mathrm{~cm}^{2} \cdot \mathrm{V}^{-1} \cdot \mathrm{s}^{-1}\right)$ due to the absence of processing residues, surface roughness atmospheric adsorbents, water, and grain boundaries [10].

van der Pauw (vdP) device measurements were performed with a custom-built setup based on a Linkam LN600P stage with environmental and temperature control. The LabVIEW program was used to control source-drain and gate voltages (Keithley 2400), multiplexing/voltage measurements (Keithley 2700 with Keithley 7709 multiplexing unit). The measurements were performed at $30{ }^{\circ} \mathrm{C}$ in a dry nitrogen environment at atmospheric pressure. All measurements were repeated several times to confirm that they were 
free of potential time-dependent measurement artifacts.

Raman maps were obtained with a Thermo Scientific DXRxi instrument with a $532 \mathrm{~nm}$ laser operating at a $180 \mathrm{~Hz}$ collection rate $(5.6 \mathrm{~ms}$ collection time), 200 scans per pixel, and a laser power of $5 \mathrm{~mW}$, with a step size of $100 \mu \mathrm{m}$ for device type $\mathrm{A}$ and $\mathrm{B}$ or $300 \mathrm{~nm}$ for device type $\mathrm{C}$. The peaks of individual spectra were fitted as described in Ref. [11]. SEM micrographs were obtained with a Zeiss Supra 60VP microscope operating with a $1 \mathrm{kV}$ acceleration voltage and a working distance of $5.0 \mathrm{~mm}$.

\section{Results and discussion}

In the dual-configuration vdP method (as further described in Figs. S1(a) and S1(b) in the Electronic Supplementary Material (ESM)), the resistance of two configurations $\mathrm{A}$ and $\mathrm{C}$ is measured as $R_{\mathrm{A}}=$ $V_{12} / I_{43}$ and $R_{C}=V_{23} / I_{14}$, where the subscripts of the I and $\mathrm{V}$ variables denote the electrodes used for passing current $I$ and measuring potential $V$, respectively (see Fig. 3(a)). The vdP sheet resistance $R_{\mathrm{vdP}}$ can be derived using the following equation [12]

$$
\mathrm{e}^{-\frac{\pi R_{\mathrm{A}}}{R_{\mathrm{VdP}}}}+\mathrm{e}^{-\frac{\pi R_{\mathrm{C}}}{R_{\mathrm{VdP}}}}=1
$$

$R_{\mathrm{A}}$ and $R_{\mathrm{C}}$ are then used to further estimate the resistance uniformity of a device, by assessing how close the resistance ratio

$$
ß=\frac{R_{\mathrm{A}}}{R_{\mathrm{C}}}
$$

is to unity (for a square device) as a function of the gate bias $\left(V_{\mathrm{G}}\right)$. Specifically, if a device has uniform current density, then the resistance ratio $R_{\mathrm{A}} / R_{\mathrm{C}}$ will exactly match that expected on the basis of the physical shape of the sample. Deviations from uniformity in a device of a certain shape can be accessed via $\beta=R_{\mathrm{A}} / R_{\mathrm{c}}$. In this way, any measured deviation of $\beta$ from its expected value is a clear indication of inhomogeneities in the sample. In other words, given a well-defined geometry, the ratio $\beta$ effectively acts as a homogeneity assessment parameter.

Figure 3 shows the sheet resistance $(R s)$ at room temperature as a function of the gate bias for three device types with $R_{\mathrm{A}}$ and $R_{\mathrm{C}}$ configurations (assuming a corresponding geometrical correction factor of $\pi / \ln (2))$, as well as the calculated sheet resistance $R_{\mathrm{vdP}}$ and $\beta$ factor. The samples shown in Fig. 3 were selected among the 50 devices investigated in this study, to represent the different behavior associated with the -- $\$$ curves of the three characteristic A, B, and C types.

In Figs. 3(a) and 3(d), type A (CVD graphene transferred to $\mathrm{Si}_{3} \mathrm{~N}_{4}$ ) is represented by devices A1 and A2, which show significant overall p-doping $\left(>2 \times 10^{13} \mathrm{~cm}^{-2}\right)$, as inferred from the position of the charge neutrality point (CNP). On the other hand, devices B1 and B2 (CVD graphene transferred to $\mathrm{SiO}_{2}$ ) in Figs. 3(e) and 3(h) show relatively low doping levels. We attribute the different doping levels of these device types to the particular transfer techniques used in each case, rather than to the specific substrates. These samples were thus selected to represent device types with different doping levels, ranging from high to low. Figures 3(i) and 3(1) show device type C (encapsulated 10 $\mu \mathrm{m} \times 10 \mu \mathrm{m}$ devices), representative of moderate n-doping levels $\left(\sim 2 \times 10^{12} \mathrm{~cm}^{-2}\right)$.

The $\beta$ curve is very sensitive to sample-to-sample variations. For the most consistent device, C2, where $R_{\mathrm{A}} \simeq R_{\mathrm{C}}$, we still observe appreciable 
differences near and on either side of the CNP. As all measurements are reproducible, we can rule out that the differences are due to $1 / f$ noise fluctuations.

To better describe the behavior observed in Fig. 3, we will employ the features present in the $\beta\left(V_{\mathrm{G}}\right)$ curves of Fig. 4(a). Any deviation from the ideal resistance ratio will be referred to as the geometrical factor $\beta_{\text {geo, }}$ whereas any variation observed as a function of $V_{G}$ will be labeled $\Delta \beta=\beta \max -\beta$ min (maximum value of $\beta$ minus minimum value of $\beta)$. For the cases where $\beta\left(V_{\mathrm{G}}\right)$ crosses unity, we use $\Delta \beta=\left(\beta_{\max }-1\right)+\left(1 / \beta_{\min }-1\right)$. Calculating $\beta$ is not equivalent to determining the effective aspect ratio of the device (except when $\beta=1$ ); as the effective aspect ratio deviates from unity, the changes in geometrical correction factors are not necessarily linear. In order to associate $\beta$ to the effective aspect ratio, we numerically evaluated $\beta$ for rectangular devices with known aspect ratio, and with the electrodes positioned at the four corners, as shown in the inset of Fig. $4(\mathrm{~b}) . R_{\mathrm{A}}$ and $R_{\mathrm{C}}$ and then $\beta$ are calculated, and the corresponding results illustrate the relationship shown in Fig. 4(b). For example, a measured $\beta=2$ is equivalent to a rectangular device with an effective aspect ratio of 1.12 , indicating that $\beta$ is very sensitive to small changes in the current paths of a device. Because $\beta$ can be calculated directly from the measurements, in the following we will continue to use $\beta$ as our figure of merit.

The three device types are significantly different in physical size, doping, mobility, etc., and have been fabricated in different ways; therefore, they are expected to exhibit different levels of homogeneity. Due to their dry (water and solvent-free) fabrication technique [10], and the encapsulation of graphene in $\mathrm{hBN}$, devices of type $\mathrm{C}$ are expected to contain the lowest levels of polymer residues, grain boundaries, and tears or folds in the graphene samples; in addition, they have not been in contact with water or other liquids, due to their fabrication technique and the encapsulation of graphene in hBN. Despite removing all these potential causes of $\Delta \beta$, a greater than $15 \%$ change in $\beta$ in the measured $V_{\mathrm{G}}$ range was observed for these devices.

A summary of the field-effect mobility values calculated for the devices in Fig. 3 is shown in Table 1. Although some devices only show a small variation in the measured mobility between the different configurations (see Fig. $3(\mathrm{k})$ ), the difference in van der Pauw mobility $\left(\mu_{\mathrm{vdP}}\right)$ between the different configurations can be larger than $15 \%$, depending on which configuration is chosen.

This finding highlights a significant issue affecting single-configuration measurements: it is the current flow and not the apparent physical dimensions of the conductor that determines $\beta$ and any resulting errors in the measured mobility. Deviations in the current path cannot be detected by a single-configuration measurement alone.

Although the vdP method provides a better representation of the overall sample properties, it tends to mask the differences between the A and C configurations. We thus suggest to use the figures of merit $(n, \mu$, gate bias required to observe CNP $\left(V_{\mathrm{CNP}}\right)$, resistance at the $\left.\mathrm{CNP}\left(R_{\mathrm{CNP}}\right)\right)$ recorded in the two configurations, alongside the vdP values and the homogeneity parameter $\beta$, to obtain a full picture of the relevant sample properties, in order to provide a baseline for quality assessment, 
inter-device comparison, and process optimization. For example, for device A1, we observed an $R_{\mathrm{CNP}}$ of $3.5 \pm 0.3 \mathrm{k} \Omega$, a hole mobility $(\mu \mathrm{h})$ of $670 \pm 54$ $\mathrm{cm}^{2} \cdot \mathrm{V}^{-1} \cdot \mathrm{s}^{-1}$, and a doping level $n=2.4 \times 10^{13} \pm 2.5 \times$ $10^{11} \mathrm{~cm}^{-2}$, with the sample uniformity described by $\beta_{\text {geo }}=1.03$ and $\Delta \beta=1.4$. Using the vdP data as well as the results from both configurations allows for a fair and accurate representation of the device uniformity, while at the same time illustrating the accuracy of the derived electrical parameters.

\subsection{Doping models}

In order to further investigate the role of doping on $\Delta \beta$, we performed finite-element calculations using Comsol software. As shown in Fig. 5(a), we divided the device area in 16 square regions, mimicking a non-constant doping landscape. Each of the squares represents graphene with a different doping level $V_{\mathrm{CNP}}$ (or equivalent value of $n$, where convenient) and with a corresponding sheet conductance defined by [13]

$$
\begin{gathered}
\sigma=n_{\text {tot }} \mu e \\
n_{\text {tot }}=\sqrt{n_{0}^{2}+n^{2}} \\
n=\frac{c_{\text {dielectric }}\left(V_{\mathrm{G}}-V_{\mathrm{CNP}}\right)}{\mathrm{e}}
\end{gathered}
$$

In Eqs. (3)-(5), $\sigma$ is the sheet conductance, $n_{\text {tot }}$ is the total sheet carrier density, $\mu$ is the carrier mobility, $e$ is the elementary charge, $n_{0}$ is the sheet carrier density at the CNP, $n$ is the sheet carrier density induced by the gate potential $V_{\mathrm{G}}$, and $C_{\text {dielectric }}$ is the capacitance per area of the oxide. The $n_{\text {tot }}$ and $\mu$ parameters were kept constant, whereas the $V_{\mathrm{CNP}}$ for each square region was set to random values confined by the upper and lower bounds of \pm $e \cdot n_{\max } / C_{\text {dielectric }}$ and a parameter sweep of $V_{\mathrm{G}}$ from -5 to $+5 \mathrm{~V}$ was conducted. We set the $C_{\text {dielectric }}$ using 300 $\mathrm{nm}$ of $\mathrm{SiO}_{2}$ and then the sweep of $V_{\mathrm{G}}$ corresponds to $n= \pm 3.6 \times 10^{11} \mathrm{~cm}^{-2}$. For each gate voltage, two electrode configurations were analyzed sequentially to extract the resistance values $R_{\mathrm{A}}$ and $R_{\mathrm{c}}$. The extent of doping uniformity of the device was altered by decreasing $n_{\max }$, in such a way that $n_{\max }=$ 0 denotes a uniform device.

Examples of the simulation output are shown as $R_{s}\left(V_{\mathrm{G}}\right)$ in Fig. 5(b), whereas the corresponding $\beta\left(V_{\mathrm{G}}\right)$ values are shown in Fig. 5(c), with solid and dashed lines corresponding to $n_{\max }=0$ and $1 \times 10^{11} \mathrm{~cm}^{-2}$, respectively. As expected, when no geometrical defects are present, $\beta_{\text {geo }}=1$ for all doping levels, and $n_{\max }=0$ led to $R_{\mathrm{A}}=R_{\mathrm{C}}=R_{\mathrm{vdP}}$, and then $\Delta \beta=0$. On the other hand, when $n_{\max }=1 \times 10^{11} \mathrm{~cm}^{-2}$, the random doping induced differences in $R_{\mathrm{A}}$ and $R_{\mathrm{C}}$ leading to a $\Delta \beta$ of 0.7 .

In order to explore the effect of doping variation on $\Delta \beta$, we varied the value of $n_{\max }$ from 0 to $1.8 \times$ $10^{11} \mathrm{~cm}^{-2}\left(2.5 \mathrm{~V}\right.$ on $\left.300-\mathrm{nm} \mathrm{SiO}_{2}\right)$ in steps of $9 \times 10^{9}$ $\mathrm{cm}^{-2}(0.125 \mathrm{~V})$. For each value of $n_{\max }$ we performed 40 iterations, using random $V_{\mathrm{CNP}}$ values for each of the 16 regions.

The average values of $\Delta \beta$ as a function of $n \max$ are shown in Fig. 6(a). The figure shows that $\Delta \beta$ increases in an approximately linear fashion as the maximum value of the doping inhomogeneity increases, and that $\Delta \beta$ is sensitive to even the smallest doping inhomogeneity, down to $10^{10} \mathrm{~cm}^{-2}$. This supports the notion that doping variations directly lead to gate-dependent variations in the electronic shape of the device, and that $\Delta \beta$ increases approximately linearly with doping inhomogeneity.

We can extract the mobility for the single A and C configurations as well as the calculated vdP mobility directly from the simulation data. The comparison of the calculated mobilities with the 
constant mobility value used as input for the finite element calculations can then be used to identify how doping inhomogeneity affects the extracted mobilities. The results are shown in Fig. 6(b) as a function of $\Delta \beta$. The figure shows that the errors in the mobilities extracted from the single-configuration measurements are significantly larger compared to the errors in $\mu_{\mathrm{vdP}}$. Moreover, even though the mobility was kept constant over the entire device, doping inhomogeneities caused the measured mobility values to be misrepresented. These data also suggest that field-effect mobility measurements become highly unreliable for larger values of $\Delta \beta$, at least for the range of doping levels explored in our simulations.

\section{$3.2 \beta_{\text {geo }}$ and $\Delta \beta$ in detail: Analysis of device A2}

In the case of device $\mathrm{A} 2$, as a result of the significant $\Delta \beta$ highlighted in Fig. 3(d), $R_{v d P}$ shows a lower apparent conductance minimum compared to the single measurements of $R_{\mathrm{A}}$ and $R_{\mathrm{C}}$ (Table 1). This further supports the conclusion that single-configuration measurements largely overestimate the carrier mobility.

In order to understand the behavior of $\beta_{\text {geo, }}$ a physical inspection of the devices can be used to identify geometric errors, such as insulating defects, cracks, tears, residual contamination, or ill-defined electrode positions. This analysis was carried out for device A2 and is shown in Fig. 7(a).

The figure shows stitched scanning electron microscopy (SEM) images of the device, which illustrate the sample morphology. Several isolated defects can be spotted in the SEM images. The insets show higher-magnification images of selected areas with similar morphology, observed for all type A devices: small sections $\left(<1 \mu \mathrm{m}^{2}\right)$ of bilayer growth, as well as extended lines that can be attributed to graphene folds caused by the difference in thermal expansion during the cooling stage of CVD growth [14]. The fold-to-fold distance is approximately 10 to $20 \mu \mathrm{m}$. The curved lines are assumed to be a result of step bunching of copper crystal edges [14]. All devices of type A were fabricated from a single CVD-grown graphene layer, so that large sample-to-sample variations are not expected. We also performed transmission electron microscopy (TEM) with selected area diffraction analysis, which showed that graphene is polycrystalline with a grain size of up to $150 \mu \mathrm{m}$ (see Fig. S2 in the ESM for details). While these grain sizes are significant, they are still small compared to the size of the device and should not contribute significantly to the measured device inhomogeneity [15], as also observed in similar transport analyses based on four-point probe measurements [16, 17]. Figure 7(b) displays a Raman map of the device, showing the intensity ratio of the $\mathrm{D}$ to $\mathrm{G}$ peaks, $I(\mathrm{D}) / I(\mathrm{G})$, which highlights areas with higher proportions of $\mathrm{sp}^{3}$ bonds, associated with defects in graphene [4]. Despite being non-uniform, the $I(\mathrm{D}) / I(\mathrm{G})$ ratio of most areas is $<0.1$, with only a few smaller areas showing higher values. Areas where no Lorentzian fit to the G-peak was possible indicate insulating defects and these areas are highlighted in white. We also note that the top and bottom edges appear significantly more defective than the vertical edges. In summary, device A2 displays considerable damage on the micrometer to millimeter scale, which is expected to affect the current flow, and cause $\beta_{\text {geo }}$ to deviate from unity, as shown in Fig. $3(d)$. 
For all devices, physical differences between individual samples can result in a current path different from that intended in the sample design. While the physical differences between samples may cause a non-uniform current path and thus a $\beta_{\text {geo }} \neq 1$, this effect is not sufficient to account for the variation of $\beta$ with the gate bias.

We then investigated the spatial variation in the doping of the samples via Raman spectroscopy maps spanning the entire devices, by observing the full width at half-maximum (FWHM) of the Raman $\mathrm{G}$ peak, $\Gamma_{\mathrm{G}}$, which is an indicator of the doping level [18]. In Fig. 7(c), higher-doped areas with $\Gamma_{\mathrm{G}}>15$ $\mathrm{cm}^{-1}$ are colored red, whereas a blue-white color indicates areas with low doping levels $\left(\Gamma_{\mathrm{G}}<15\right.$ $\mathrm{cm}^{-1}$ ). The figure reveals some of the line-like features that were visible in the SEM micrograph of Fig. $7(\mathrm{a})$, but not in the $I(\mathrm{D}) / I(\mathrm{G})$ map of Fig. $7(\mathrm{~b})$. These features can thus be attributed to doping rather than defects, which may also lead to variations in $\Delta \beta$, but should exhibit a dependence on the gate voltage as well.

Variations in the position of the CNP have been explored in graphene devices before, and it was found that the so-called charge puddles give rise to variations in $\beta$ for small enough device sizes. However, the areas of these charge puddles have been reported to reach several tens of square nanometers [15]. In addition, their effects have been reported to average out and vanish for samples with device areas larger than approximately $1 \mu \mathrm{m} \times$ $1 \mu \mathrm{m}$ [15], while even our smallest devices exceed this area by two orders of magnitude. Thus, any effect due to charge puddles, as previously reported, can be neglected for the present devices.
Finite element simulations were used to simulate the gate dependence of device A2 as described above, based on the Raman maps shown in Fig. 7 . The map of isolated defects extracted from Figs. $5(a)-5(b)$ was used to define the overall device shape, and the approximate doping landscape was derived from the Raman map shown in Fig. 7(c). Figure 7(c) gives $50 \times 50$ pixels in the device region of $5 \mathrm{~mm} \times 5 \mathrm{~mm}$, and three areas with different doping levels are identified by white $\left(<11 \mathrm{~cm}^{-1}\right)$, blue (11-15 $\left.\mathrm{cm}^{-1}\right)$, and red $\left(>15 \mathrm{~cm}^{-1}\right)$ colors. The mobility was kept constant at the measured value $\mu_{\mathrm{vdP}}=438 \mathrm{~cm}^{2} \cdot \mathrm{V}^{-1} \cdot \mathrm{s}^{-1}$, and only the doping values were allowed to vary.

The simulated data converged to approximate the experimental data, as shown in Fig. 8. The good agreement with the experimental data suggests that the $\Gamma_{\mathrm{G}}$ map provides a reasonably accurate representation of the doping landscape in device $\mathrm{A} 2$, when suitable values for the white region $\left(V_{\mathrm{CNP}}\right.$ $=65 \mathrm{~V})$, blue region $\left(V_{\mathrm{CNP}}=67 \mathrm{~V}\right)$, and red region $\left(V_{\mathrm{CNP}}=94 \mathrm{~V}\right)$ are used. Considering the low resolution of the doping map and the crude assumption that the doping of the three areas was constant, the agreement is very reasonable. In order to determine $\beta_{\text {geo, }}$ we set the doping level of each region to a constant value and obtained $\beta_{\text {geo }}=1.94$, slightly lower than the $\beta_{\text {geo }}$ of 2.21 extracted from the experimental data, which represents a good agreement. The gate dependence of inhomogeneous graphene devices simulated using Raman maps as input for the doping variation is consistent with the experimental observations discussed above, which indicated that the inhomogeneous CNP position is the primary cause of $\Delta \zeta$ variations for the systems examined here. 
A multitude of factors contribute to the local variations in the CNP, such as (but not limited to) surface water [19], adsorbed gases [20], rips/tears [21], folds [22], sections of multilayer graphene [22], polymer residues [13], grain boundary distributions [22], variation in stress [23], strain [13], etc.; however, a specific investigation of these factors is beyond the scope of this study. For certain types of devices, it is also possible that variations in carrier mobility may have a more significant effect on the sheet conductance than doping inhomogeneities [24], however, we found that this represents at most a secondary effect for the present systems.

The $\beta_{\text {geo }}$ and $\Delta \beta$ measurements are straightforward and accessible to anyone with a four-point measurement system available. The van der Pauw correction can be used for square devices, Hall bars, or other arbitrarily shaped devices, implying that the present analysis method can be applied to other systems by simply switching voltage and current inputs between measurements. Obviously, an automated multiplexing contact system could greatly improve the speed and convenience of this procedure, allowing direct measurements of the gate dependence of $\Delta \beta$.

\section{Conclusions}

The electrical aspect ratio $\beta$ of graphene devices was measured for 50 devices of three different types. All devices showed variations in $\beta$ as a function of the gate bias. Simple finite element simulations suggest that a major cause of the $\beta$ variability in the present samples is represented by doping inhomogeneities. Deviations of $\beta_{\text {geo }}$ from its expected value for a certain geometrical shape can be attributed to insulating defects, which can be identified by a physical inspection of the sample and/or Raman maps to determine their graphene coverage, and can be further confirmed by the finite element simulations.

Finite element simulations show that the dual-configuration vdP method provides greater accuracy over single-configuration method for investigating graphene devices. The present results suggest that $\beta$ is an important figure of merit for graphene devices and for other materials where similar inhomogeneities are present. Single-configuration measurements can very easily yield inaccurate or incorrect sheet resistance values, in turn leading to considerable inaccuracies in the calculation of other key figures of merit (carrier mobility, carrier density, and sheet resistance). On the other hand, if the electrical parameters are obtained through dual-configuration vdP measurements, then $\beta$ can be easily determined and any errors identified. These findings thus suggest that the uncertainties in the measurements of device properties should be estimated from differences in the $\mu, R_{\max }$ and CNP parameters determined for $\mathrm{A}$ and $C$ configurations, since this additional information reveals the degree of variability of each parameter.

\section{Acknowledgements}

We would like to thank the Nanocarbon group at DTU Nanotech for valuable discussions as well as the staff at DTU Danchip for technical and fabrication advice. For funding we would like to acknowledge DNRF103 CNG, HC Ørsteds foundation, Villum Fonden project (No.VKR023117), the DA-GATE project (No. 
12-131827), and EC Graphene FET Flagship contract number 604391 as well as H2020 European projects (Nos. 692527 and 688225).

Electronic Supplementary Material: Supplementary material (Dual configuration, and TEM techniques) is available in the online version of this article at https://doi.org/10.1007/s12274-017-1570-y. 
Table 1 Summary of hole mobilities $\left(\mathrm{cm}^{2} \cdot \mathrm{V}^{-1} \cdot \mathrm{s}^{-1}\right)$ and $\beta$ values for the devices shown in Fig. 3

\begin{tabular}{lllllll}
\hline Device & $\mu_{\mathrm{vdP}}$ & $\mu_{\mathrm{A}}$ & $\mu_{\mathrm{C}}$ & $\mu_{\mathrm{A}} / \mu_{\mathrm{C}}$ & $\Delta \beta$ & $\beta_{\text {geo }}$ \\
\hline A1 & 670 & 636 & 690 & 1.09 & 1.4 & 1.03 \\
$\mathrm{~A} 2$ & 438 & 994 & 160 & 6.21 & 5.3 & 2.21 \\
B1 & 483 & 513 & 448 & 1.14 & 0.62 & 2.27 \\
B2 & 86 & 61 & 146 & 2.39 & 0.55 & 2.1 \\
C1 & 23,138 & 20,468 & 23,292 & 1.14 & 0.20 & 1.18 \\
C2 & 37,110 & 35,760 & 30,880 & 1.15 & 0.20 & 1.07 \\
\hline
\end{tabular}

a)

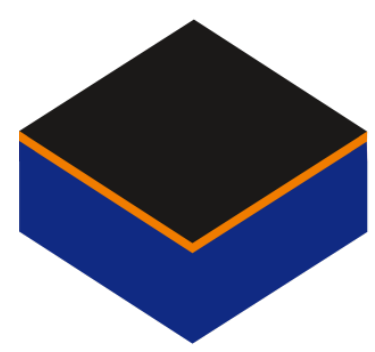

Graphene b)

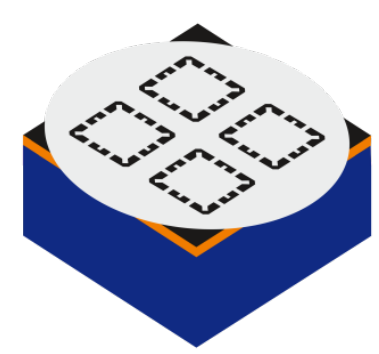

c)

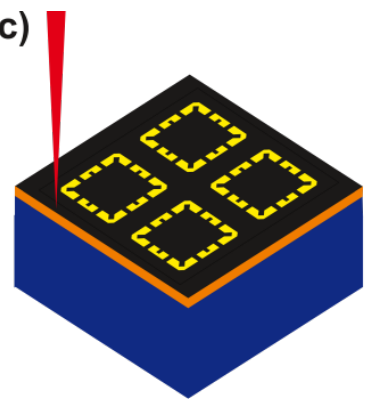

d)

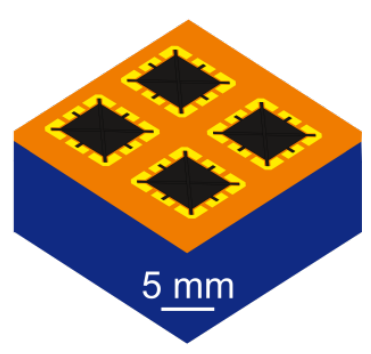

$\mathrm{SiO}_{2}$ or $\mathrm{Si}_{3} \mathrm{~N}_{4} \quad \mathrm{Si} \quad$ Shadow Mask Chrome/Gold $\quad$ Pulsed laser

Figure 1 Illustration of laser ablation-based device fabrication [8]. (a) Single-layer graphene is deposited onto a silicon substrate with a silicon nitride or silicon dioxide dielectric. (b) Metal electrodes are defined via a shadow mask. (c) A pulsed laser with $\lambda=1,064 \mathrm{~nm}$ ablates graphene without damaging the substrate. (d) The devices are ready for measurement. 


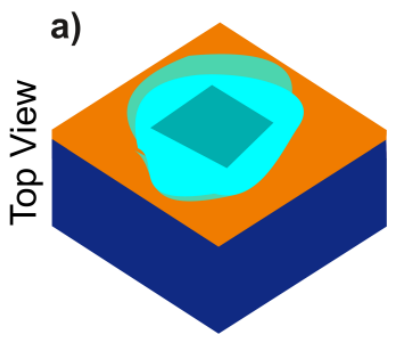

b)
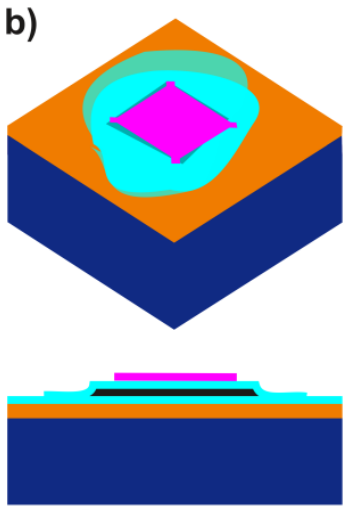

Graphene c)
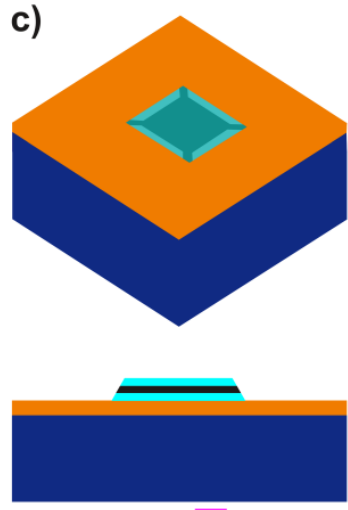

EBL resist d)
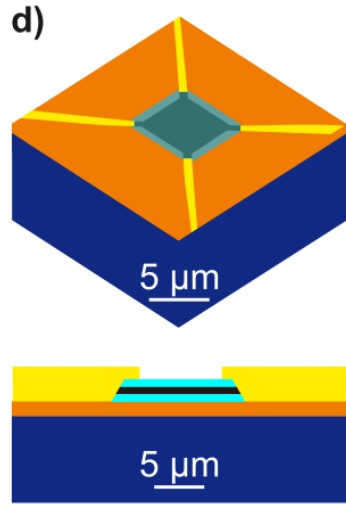

Chrome/Gold

Figure 2 Illustration of the fabrication of encapsulated graphene devices [10]. (a) Stack consisting of top layer hBN, middle layer graphene, bottom layer hBN, with graphene fully encapsulated. (b) EBL is used to define an etch mask in PMMA. (c) Selective etching into a van der Pauw geometry. (d) Metal contacts are defined via a standard EBL process.
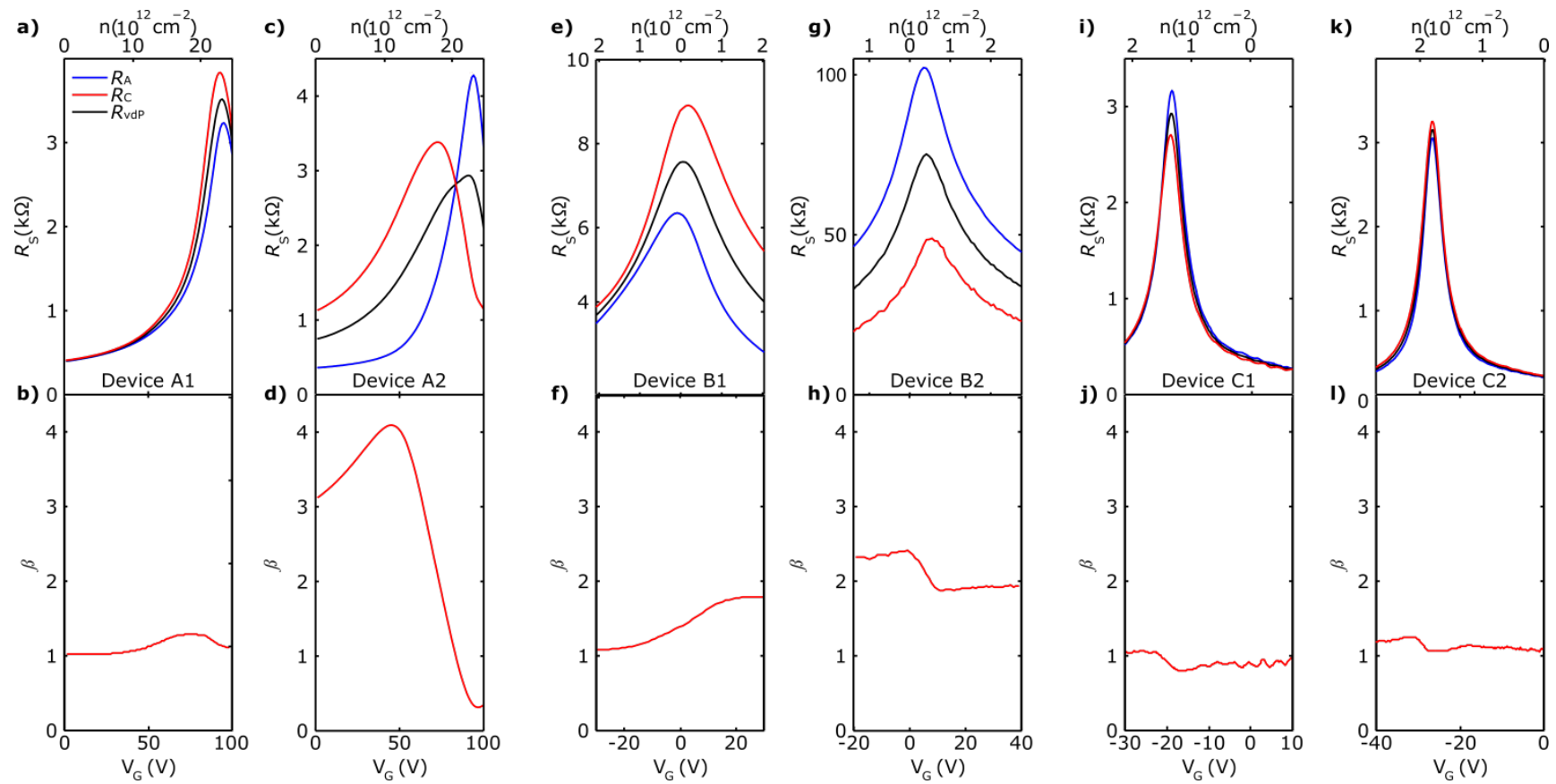

Figure 3 Gate bias $\left(V_{\mathrm{G}}\right)$ dependence of the samples shown in Figs. 7 and 8. ((a), (c), (e), (g), (i), (k)): sheet resistance $\left(R_{\mathrm{s}}\right)$ of configurations A (blue) and C (red), and calculated sheet resistance $R_{\mathrm{vdP}}$ (black) of the devices. ((b), (d), (f), (h), (j), (l)): ratio ( $\beta$ ) of the resistances measured for $\mathrm{A}$ and $\mathrm{C}$ configurations. 

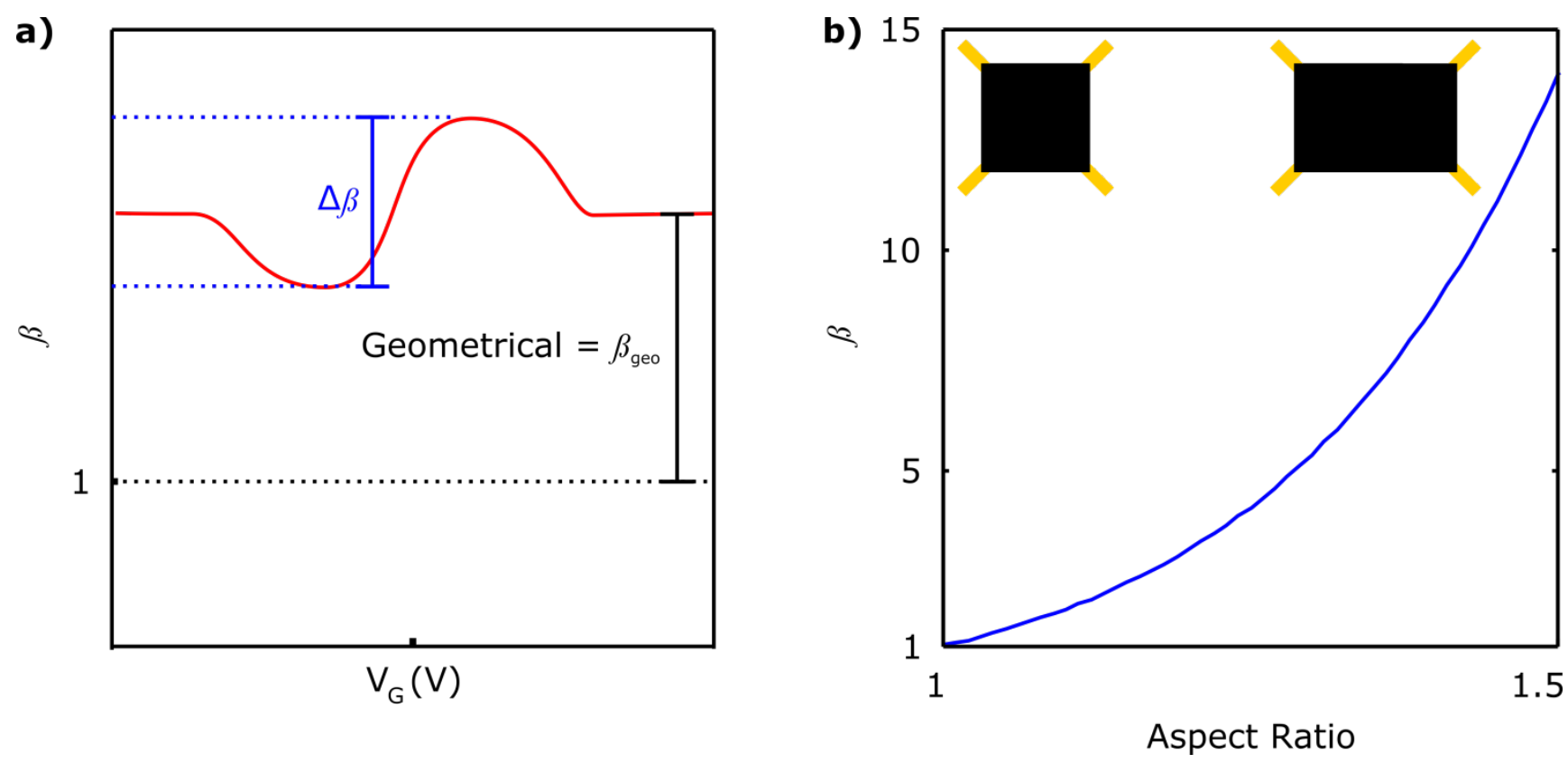

Figure 4 (a) Schematic showing the two components of $\beta$. The geometrical difference $\beta_{\text {geo }}$ denotes any deviation from $\beta=1$, whereas $\Delta \beta$ represents the $\beta$ change due to the gate bias. (b) Calculated relationship between physical aspect ratio and $\beta$ for rectangular devices with electrodes positioned in the corners. The inset shows example devices with aspect ratio 1 (left) and 1.5 (right).

a)

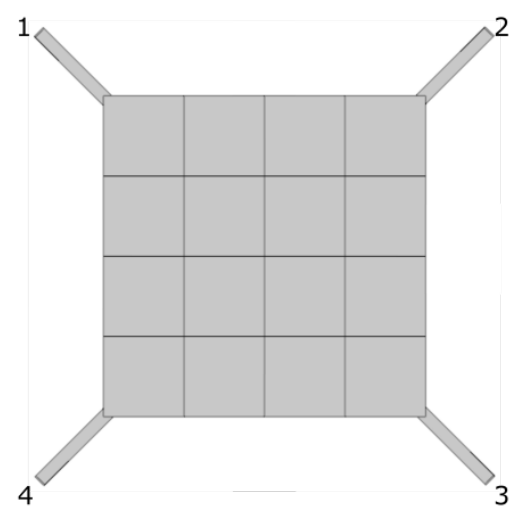

b)

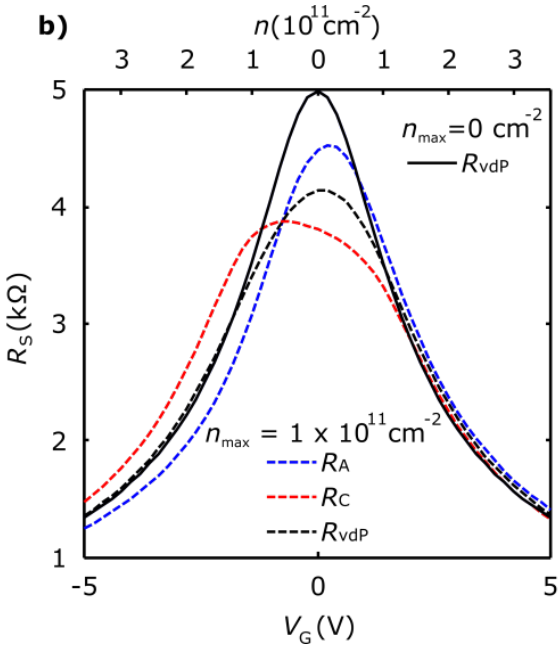

c)

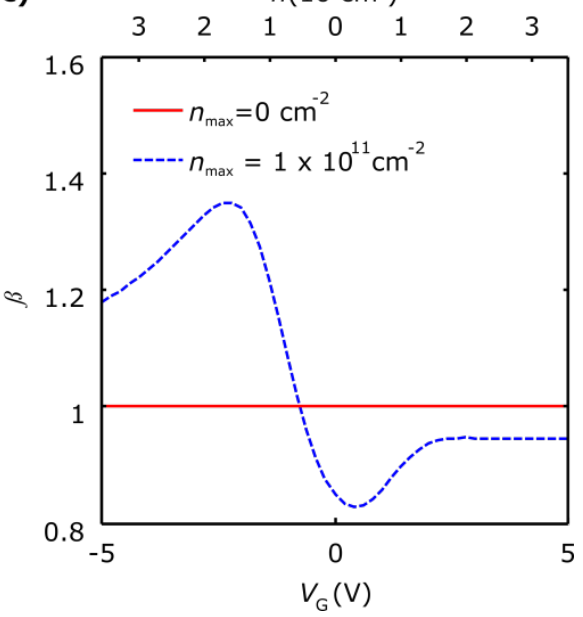


Figure 5 Overview of finite element simulations for a generic square device in van der Pauw geometry. (a) Device geometry showing the electrode positions $\left(R_{\mathrm{A}}=V_{12} / I_{43}\right.$ and $\left.R_{\mathrm{C}}=V_{23} / I_{14}\right)$. (b) Examples of gate sweeps generated for a uniform (solid line) and an inhomogeneous (dashed line) device. (c) $\beta$ curves generated from Fig. 5(b).
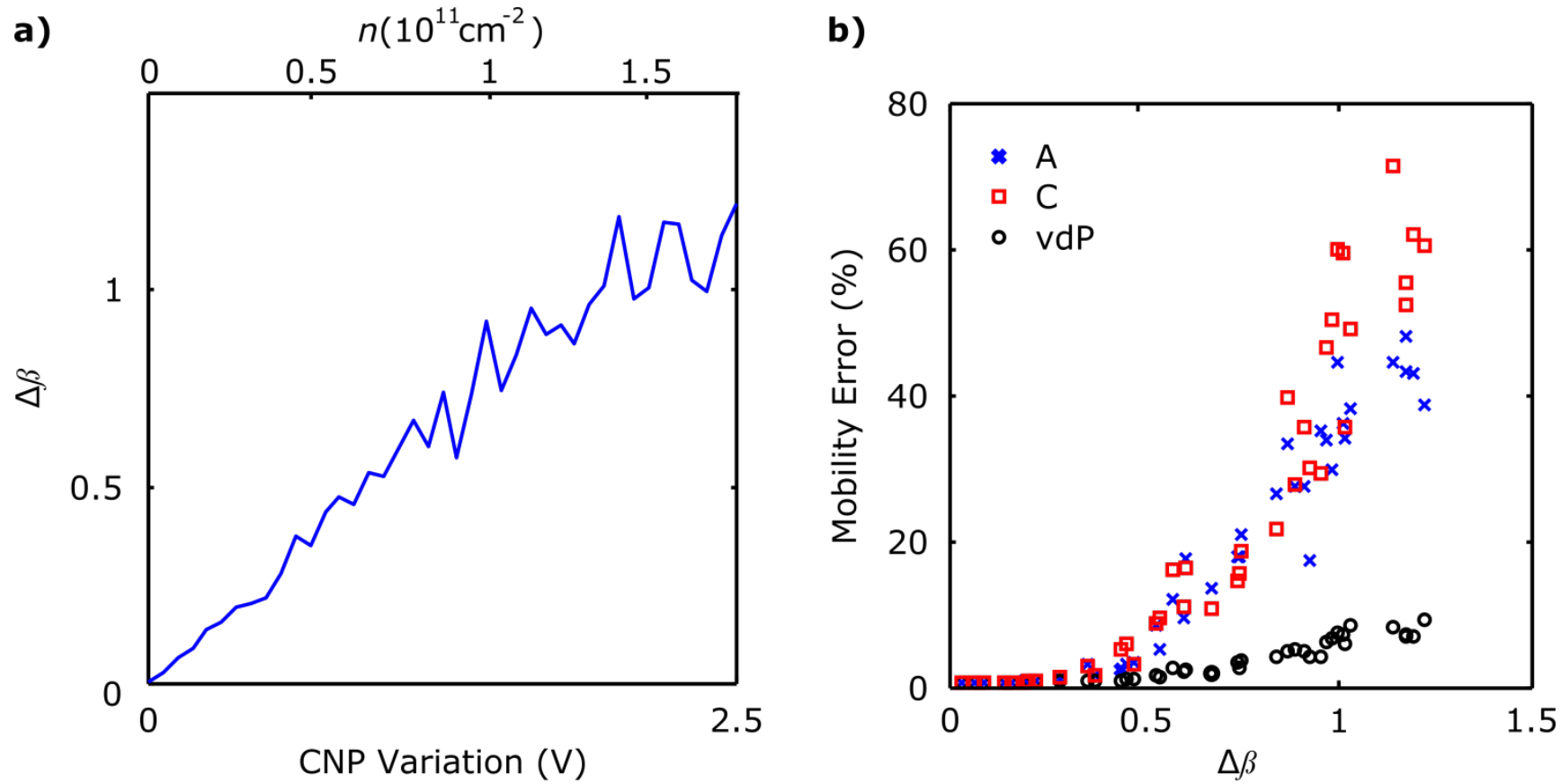

Figure 6 (a) $\Delta \beta$ variation as a function of doping homogeneity. (b) Error in calculated mobility for A configuration, C configuration, and vdP.
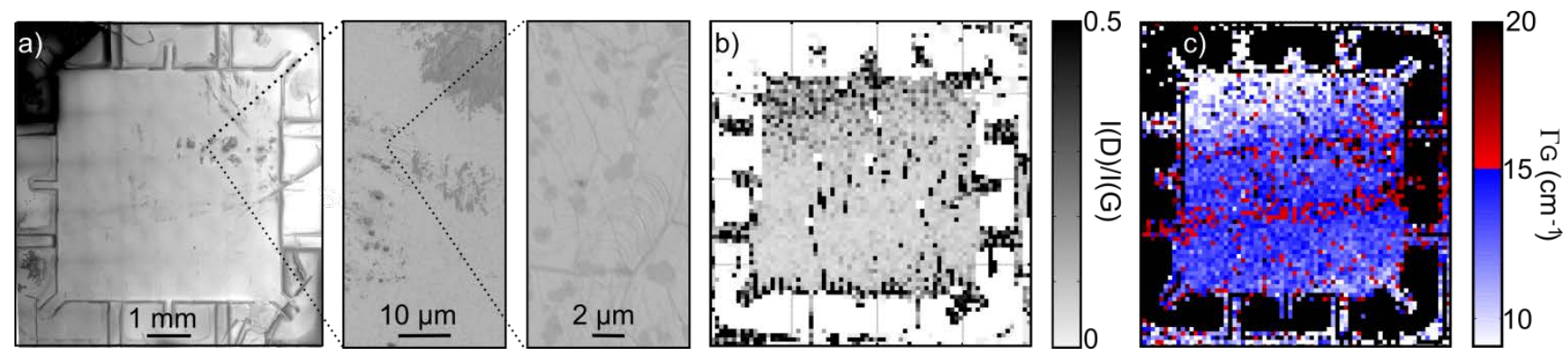

Figure 7 Images of device A2. (a) Stitched scanning electron micrograph with enlarged view of damaged area. (b) Raman map obtained from 5,625 spectra showing the intensity ratio of $G$ to D peaks $(I(D) / I(G))$, with areas for which the Raman peaks could not be fitted 
highlighted in white. (c) Raman map obtained from 5,625 spectra showing the FWHM of the $\mathrm{G}$ peak $\left(\Gamma_{\mathrm{G}}\right)$, with areas for which the Raman peaks could not be fitted highlighted in black.

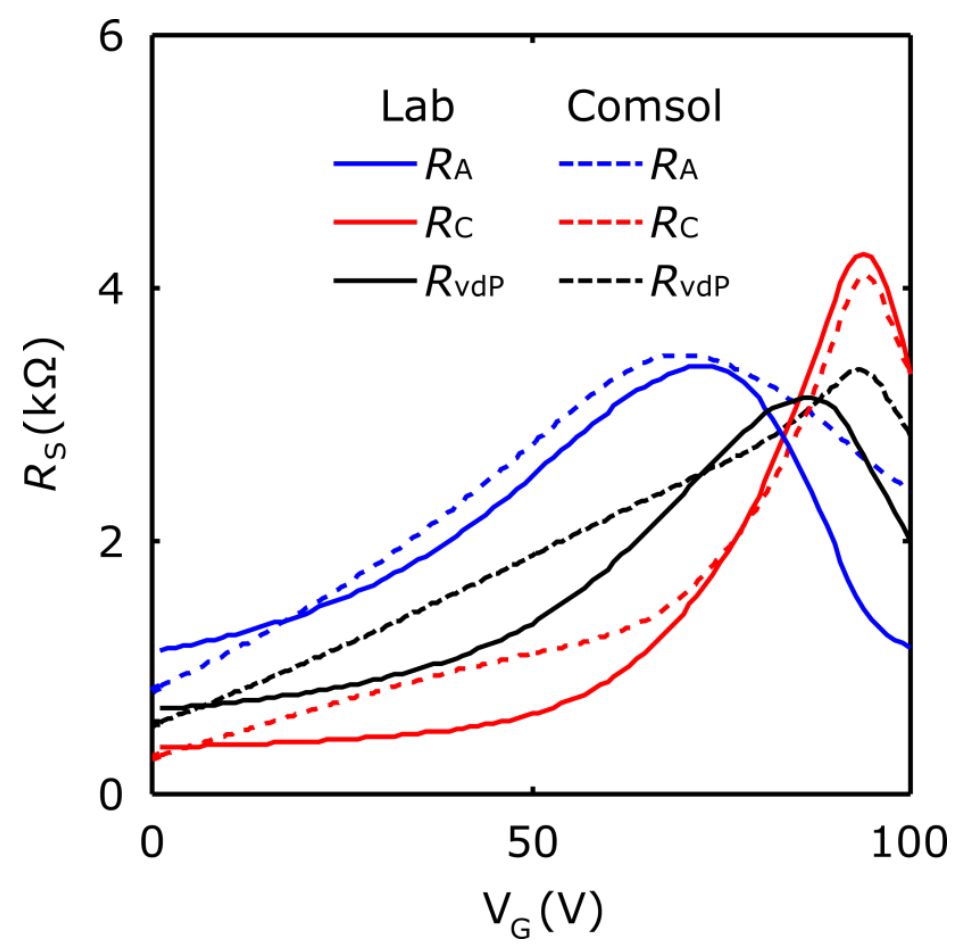

Figure 8 Device A2: Comparison of electrical measurements (solid lines) and simulated gate data (dashed lines) obtained from the Comsol software using Raman maps as input for insulating defects and doping locations. 